\title{
Ultrasound assisted synthesis of 2, 4-substituted 1, 5- benzothiazepine derivatives
}

Mangesh S. Ghodke ${ }^{a^{*}}$, Anna Pratima G. Nikalje ${ }^{\mathrm{b}}$, Suvarna A.Wagh ${ }^{\mathrm{c}}$, Julio A Seijas Vázquez ${ }^{\mathrm{d}}$

a. Department Of Pharmaceutical Chemistry, R. C. Patel Institute Of Pharmaceutical Education \& Research, Shirpur-425405, Dhule (M.S.) India

b. Wilson college, Girgaon Chawpatty,Mumbai 400007 , Maharashtra, India

c. MVP's Institute of Pharmaceutical Sciences, Adgoan, Nashik-422003 (M.S.) India.

d. Departamento de Química Orgánica, Facultad de Ciencias, Universidad of Santiago De Compostela, Alfonso X el Sabio, Lugo 27002, Spain

\begin{abstract}
A series of 15 novel derivatives of Synthesis of 2, 4-substituted 1, 5- benzothiazepine 5(a-I) were synthesized in good yield from the key compound 1, 3-substituted-prop-2-en-1-one 3(a-l), called chalcone, with 2-aminothiophenol $(0.01 \mathrm{~mol})$ and pinch of zirconium oxychloride and solvent dimethyl formamide (DMF), by using ultra-sonication as one of the green chemistry tools. The 1,5benzothiazepine scaffold has several biological activity such as vasodilator, antiarrythmic antagonists of several G-protein coupled receptors such as cholecystokinin (CCK) receptor, antiulcer activity, anticonvulsant activity.
\end{abstract}

Keywords: Green chemistry; Ultra-sonication; Chalcone 


\section{Introduction}

Synthesis of nitrogen and sulphur containing heterocycles is of current interest because of their useful, broad spectrum biological activities. The heterocycle such as 1,5-benzothiazepine have gained attention of medicinal chemists due to their use as drugs and medicaments in a large number of diseases.

1,5-benzothiazepine derivatives are well known on account of their wide synthetic applications and biological/pharmaceutical values. The 1,5-benzothiazepine scaffold has been used as cardiovascular modulator ${ }^{1}$ such as vasodilator ${ }^{2,3}$ and antiarrythmic ${ }^{4}$ antagonists of several G-protein coupled receptors such as cholecystokinin $(\mathrm{CCK})$ receptor $^{5}$, antiulcer activity ${ }^{6}$, spasmolytic activities $^{7-}$ ${ }^{8}$, ACE inhibitors ${ }^{9}$, the angiotensin II receptor (ACE) inhibitors ${ }^{10}$. Benzothiazepines are bioisosters of benzodiazepines. The recent succesful exploitation of 1,5-benzothiazepine type of compounds such as diltiaze, thiazepam, chlordiazepoxide, diazepam and chlobazam as potential CNS, cardiovascular and psychopharmacological agents, prompted us to synthesis a new class of potential 1,5-benzothiazepine with heteyl moiety like indole, alipatic and aromatic ketones.

As the process of drug discovery is very time consuming, tedious and expensive, therefore, the efforts are made to minimize the duration of reaction time, enhance the yield, minimize or avoid use of solvents, especially the hazardous solvents. The green chemistry tools such as Ultrsound synthesizer, solvent-free reactions are used so as to avoid pollution of surroundings.

Sono-chemistry is the application of ultrasound technique has undergone very intensive research and development in the last 15-20 years to carry out chemical transformation. Ultrasound offers potential for cleaner reactions called green protocol keeping in mind to protect environment clean and green through improved product yields and selectivity towards desired product, reduce the time of completion of reaction [11-13] and enhanced ease of product recovery.

Ultrasound-promoted synthesis has various advantages over conventional synthesis techniques such as reactions were carried at room temperature and require much less time for completion hence saves time and electricity, highly accelerated reaction rate, requires use of very less amount of solvents, shortened work-up procedure. practically good yield of product, simple instrument with control on reaction parameters, and most important is eco- friendly neat and clean synthetic protocol.

In this research work we are reporting the synthesis of 2, 4-substituted 1, 5- benzothiazepine 5(a-l) derivatives in excellent yield using eco- friendly, prompt and suitable ultrasound-assisted green chemistry protocol. 


\section{Results and Discussion}

\subsection{Chemistry}

Herein, we are reporting the synthesis $\mathbf{2 ,}$ 4-substituted 1, 5- benzothiazepine 5(a-l) as illustrated in

\section{Scheme 1}<smiles>[R]C(C)=O</smiles>

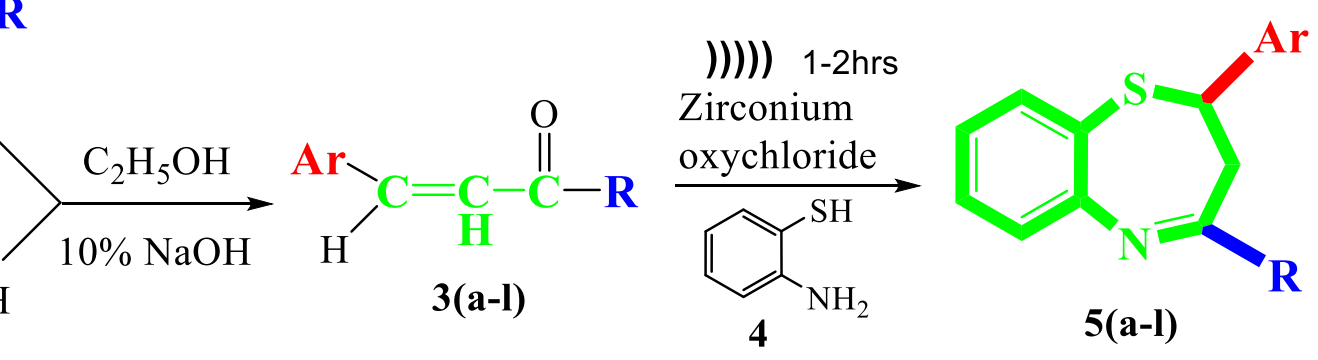<smiles>O=CC([17OH])[18F]</smiles>

Where Ar= 1) Aromatic<smiles>[R][X]1ccc(C)c1</smiles>

2) Heteryl

$$
\mathrm{R}^{\prime}=\mathrm{OCH}_{3}, \mathrm{OH}, \mathrm{Cl}, \mathrm{F}, \mathrm{OC}_{2} \mathrm{H}_{5}, \mathrm{CH}_{3}
$$$$
\mathbf{R}^{\prime}=-\mathrm{CH}_{3},-\mathrm{C}_{2} \mathrm{H}_{5}
$$

\section{Scheme I}

A novel series of 2, 4-substituted-1, 5-benzothiazepines were efficiently synthesized under ultrasound irradiation. Here Step I includes synthesis of 1, 3-substituted-prop-2-en-1-one 3(a-l) by reacting substituted aromatic aldehydes with ketone such as dimethyl ketone and ethyl methyl ketone. Physical characterization data of $3 \mathbf{a}-\mathbf{j}$ were shown in Table1.

Table1. Physical characterization of 1, 3-substituted-prop-2-en-1-one 3(a-l)

\begin{tabular}{|c|c|c|c|c|c|c|}
\hline Code & $\mathbf{R}$ & $\mathbf{A r}$ & Mol. Formula & $\begin{array}{l}\text { Mol. } \\
\text { Wt. }\end{array}$ & $\begin{array}{l}\text { Yield }(\%) \\
\text { M.P }\left({ }^{\circ} \mathrm{C}\right)\end{array}$ & Rf Value \\
\hline $3 \mathbf{a}$ & $-\mathrm{CH}_{3}$ & & $\mathrm{C}_{10} \mathrm{H}_{10} \mathrm{O}$ & 146.19 & $90(120)$ & 0.61 \\
\hline $3 \mathbf{b}$ & $-\mathrm{C}_{2} \mathrm{H}_{5}$ & & $\mathrm{C}_{11} \mathrm{H}_{12} \mathrm{O}$ & 160.22 & $50(65)$ & 0.70 \\
\hline $3 c$ & $-\mathrm{CH}_{3}$ & & $\mathrm{C}_{10} \mathrm{H}_{10} \mathrm{O}_{2}$ & 162.19 & $65(72)$ & 0.54 \\
\hline 3d & $-\mathrm{C}_{2} \mathrm{H}_{5}$ & & $\mathrm{C}_{11} \mathrm{H}_{12} \mathrm{O}_{2}$ & 176.22 & $56(75)$ & 0.59 \\
\hline $3 e$ & $-\mathrm{CH}_{3}$ & & $\mathrm{C}_{11} \mathrm{H}_{12} \mathrm{O}_{3}$ & 192.21 & $60(70)$ & 0.64 \\
\hline $3 f$ & $-\mathrm{C}_{2} \mathrm{H}_{5}$ & & $\mathrm{C}_{12} \mathrm{H}_{14} \mathrm{O}_{3}$ & 206.24 & $70(90)$ & 0.62 \\
\hline
\end{tabular}




\begin{tabular}{|c|c|c|c|c|c|c|}
\hline $3 g$ & $-\mathrm{CH}_{3}$ & $\mathrm{OCH}_{3}$ & $\mathrm{C}_{13} \mathrm{H}_{16} \mathrm{O}_{4}$ & 236.27 & $67(65)$ & 0.508 \\
\hline $3 h$ & $-\mathrm{C}_{2} \mathrm{H}_{5}$ & & $\mathrm{C}_{14} \mathrm{H}_{18} \mathrm{O}_{4}$ & 250.29 & $80(90)$ & 0.62 \\
\hline $3 \mathbf{i}$ & $-\mathrm{CH}_{3}$ & & $\mathrm{C}_{11} \mathrm{H}_{12} \mathrm{O}$ & 160.22 & $65(80)$ & 0.40 \\
\hline $3 \mathbf{j}$ & $-\mathrm{C}_{2} \mathrm{H}_{5}$ & & $\mathrm{C}_{12} \mathrm{H}_{14} \mathrm{O}$ & 174.24 & $56(85)$ & 0.66 \\
\hline $3 \mathbf{k}$ & $-\mathrm{CH}_{3}$ & & $\mathrm{C}_{10} \mathrm{H}_{9} \mathrm{ClO}$ & 180.63 & $60(90)$ & 0.45 \\
\hline 31 & $-\mathrm{C}_{2} \mathrm{H}_{5}$ & & $\mathrm{C}_{11} \mathrm{H}_{11} \mathrm{ClO}$ & 194.66 & $75(80)$ & 0.56 \\
\hline
\end{tabular}

Step II involved synthesis of 2, 4-substituted 1, 5- benzothiazepine 5(a-l) when compounds 3(a-1) reacted with ortho-amino thiophenol using a pinch of zirconium oxychloride as a catalyst under ultrasound irradiation using solid probe type synthesizer at room temperature. Physical characterization data of were shown in Table2.

Table2. Physical characterization of 2, 4-substituted 1, 5- benzothiazepine 5(a-1)

\begin{tabular}{|c|c|c|c|c|c|c|c|}
\hline Code & $\mathbf{R}$ & Ar & $\begin{array}{c}\text { Mol. } \\
\text { Formula }\end{array}$ & $\begin{array}{l}\text { Mol. } \\
\text { Wt. }\end{array}$ & $\begin{array}{c}\text { Time } \\
\text { In } \\
\text { min. }\end{array}$ & $\begin{array}{l}\text { Yield }(\%) / \\
\text { M.P }\left({ }^{\circ} \mathrm{C}\right) *\end{array}$ & $\begin{array}{c}\text { Rf } \\
\text { Value } * *\end{array}$ \\
\hline $\mathbf{5 a}$ & $-\mathrm{CH}_{3}$ & & $\mathrm{C}_{16} \mathrm{H}_{15} \mathrm{NS}$ & 253.36 & $45-50$ & $90(122)$ & 0.36 \\
\hline $5 \mathbf{b}$ & $-\mathrm{C}_{2} \mathrm{H}_{5}$ & & $\mathrm{C}_{17} \mathrm{H}_{17} \mathrm{NS}$ & 267.39 & $55-58$ & $50(125)$ & 0.72 \\
\hline $5 c$ & $-\mathrm{CH}_{3}$ & & $\mathrm{C}_{16} \mathrm{H}_{15} \mathrm{NOS}$ & 269.36 & $62-65$ & $65 /(134)$ & 0.71 \\
\hline $5 d$ & $-\mathrm{C}_{2} \mathrm{H}_{5}$ & & C17H17NOS & 283.39 & $60-65$ & $56(137)$ & 0.83 \\
\hline $5 e$ & $-\mathrm{CH}_{3}$ & & $\mathrm{C}_{17} \mathrm{H}_{17} \mathrm{NO}_{2} \mathrm{~S}$ & 299.39 & $70-74$ & $60(140)$ & 0.54 \\
\hline
\end{tabular}




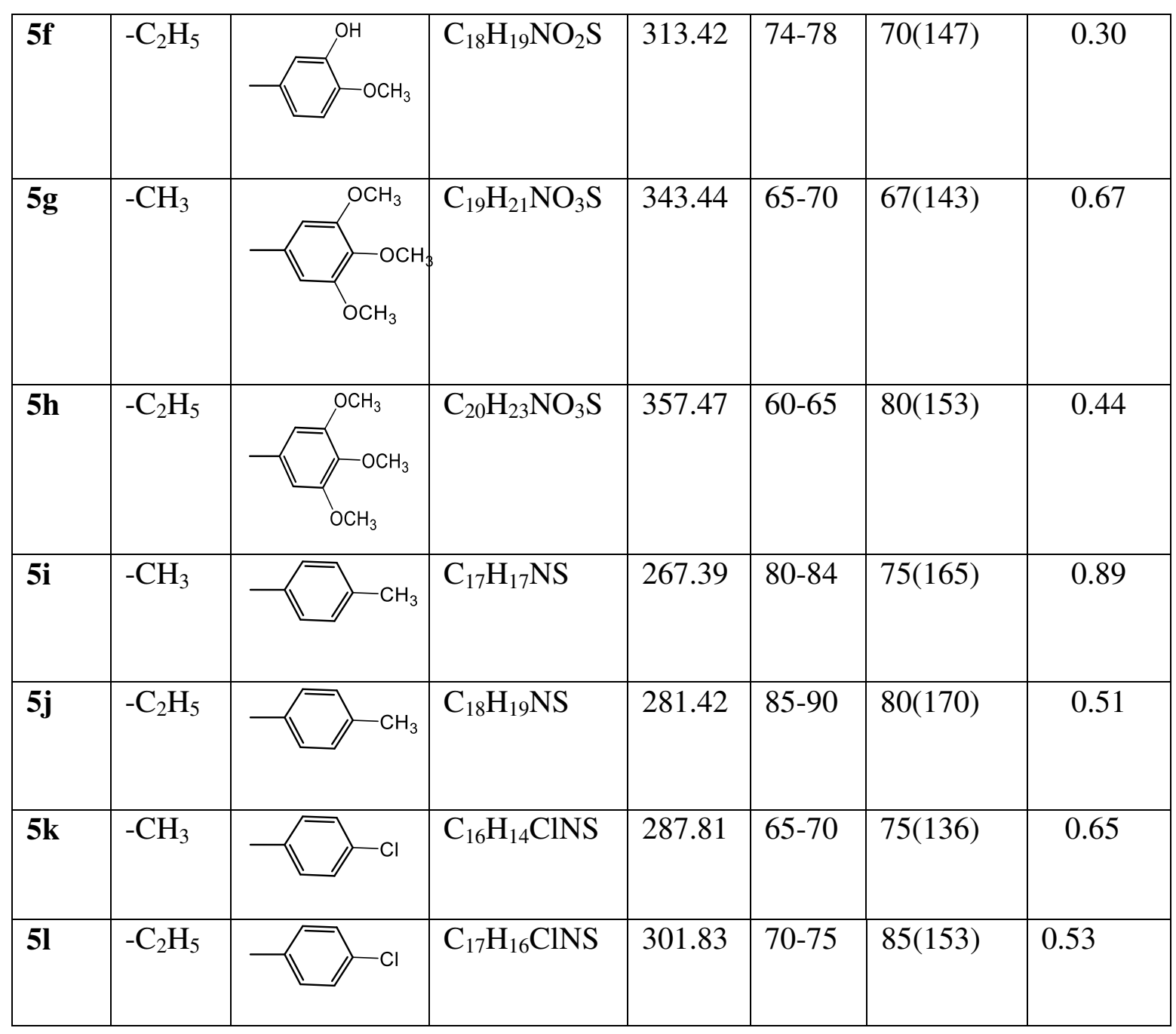

\section{Methods}

\subsection{General Information}

All the chemicals used for synthesis were procured from Merck (Mumbai, Maharashtra, India),

Sigma (Mumbai), HiMedia (Mumbai) or Qualigens (Mumbai) and used without further purification. The progress of each reaction was monitored by ascending thin layer chromatography (TLC) using pre-coated silica gel F254 aluminum TLC sheets (Merck) and the spots were visualized by UV light and iodine vapors. Elemental analyses (C, H, and N) were done with a FLASHEA 112 Shimadzu' analyzer (Mumbai) and all analyses were consistent (within 0.4\%) with theoretical values. Infrared (IR) spectra were recorded on a PS 4000 FTIR (JASCO, Tokyo, Japan) using KBr pellets. 1H- and 13C-NMR (200 MHz) spectra were recorded on a ACF 200 spectrometer (Bruker, Billerica, MA, USA) fitted with an Aspect 3000 computer and all the chemical shifts (ppm) were referred to internal TMS for 1H and chloroform-d for 13C-NMR.1H-NMR data are reported in the order of chemical shift, number of protons. For ultrasound irradiation Vibra cell VCX-500 with solid probe was used (Sonics, Newtown, CT, USA). 


\subsection{Experimental Section}

\subsubsection{Step I: Synthesis of 1, 3-substituted-prop-2-en-1-one ${ }^{14} 3(\mathrm{a}-\mathrm{l})$}

These chalcone analogues were prepared by stirring aldehyde $(0.01 \mathrm{~mol}$.) and various ketones $(0.01$ mol.) in ethanol containing $10 \% \mathrm{NaOH}$ in separate flasks. The reaction mixture was kept aside for 24 hrs and then it was poured on crushed ice and was acidified with dil. $\mathrm{HCl}$. The crude product thus obtained was filtered, dried and recrystallized with ethanol.

\subsubsection{Step II: Synthesis of 2, 4-substituted 1, 5- benzothiazepine ${ }^{15,16,17} 5(\mathrm{a}-\mathrm{l})$}

In a beaker, compound 16(a-l) $(0.01 \mathrm{~mol})$, 2-aminothiophenol $(0.01 \mathrm{~mol} 1.27 \mathrm{ml})$ and pinch of zirconium oxychloride were taken in DMF $(20 \mathrm{ml})$. The reaction mixture was kept inside the acoustic box having solid probe type Ultrasonic processor for about 1-2 hours at room temperature. After completion of the reaction (monitored by TLC), the mixture was poured into ice cold water.

\section{4-methyl-2-(4-hydroxy phenyl)-2,3-dihydrobenzothiazepine (5c)}

IR (KBr): $v=3479 \mathrm{~cm}^{-1}(\mathrm{OH}), 3020 \mathrm{~cm}^{-1}($ aromatic $-\mathrm{CH}) 2900,2802\left(-\mathrm{CH}_{2} \mathrm{CH}_{3}\right), 1592(\mathrm{C}=\mathrm{N}), 675$ (C-S). ${ }^{1} \mathrm{H} \mathrm{NMR}\left(\mathrm{CDCl}_{3}\right): \delta \mathrm{ppm}=1.3(\mathrm{~s}, 3 \mathrm{H}), 3.4(\mathrm{dd}, 2 \mathrm{H}), 4.5(\mathrm{dd}, 1 \mathrm{H}), 6.5-8(\mathrm{~m}, 12 \mathrm{H}, \mathrm{ArH}), 8.2(\mathrm{~s}$, aromatic $\mathrm{OH}),{ }^{13} \mathrm{CNMR}\left(\mathrm{CDCl}_{3}\right) \delta \mathrm{ppm}: 11.7,26.8,37.8,50.5,115.9,117.0,125.8,127.4,129.1$, 133.4, 136.1, 151.4, 156.9. Mass (m/z): 283. Anal. Calcd. For $\mathrm{C}_{19} \mathrm{H}_{18} \mathrm{~N}_{2} \mathrm{~S}: \mathrm{C}, 72.04 ; \mathrm{H}, 6.05 ; \mathrm{N}, 4.94$. Found: C, 71.02; H, 5.50; N, 4.5.

\section{2-(3-hydroxy-4-methoxyphenyl)-4-ethyl-2,3-dihydro-1,5-benzothiazepine (5f)}

IR (KBr): $v=3328 \mathrm{~cm}^{-1}(\mathrm{OH}), 3025 \mathrm{~cm}^{-1}$ (aromatic $\left.-\mathrm{CH}\right) 2850,\left(-\mathrm{CH}_{2} \mathrm{CH}_{3}\right), 1580(\mathrm{C}=\mathrm{N}), 670(\mathrm{C}-\mathrm{S})$. ${ }^{1} \mathrm{H} \mathrm{NMR}\left(\mathrm{CDCl}_{3}\right): \delta \mathrm{ppm}=1.2(\mathrm{~s}, 3 \mathrm{H}), 3.3(\mathrm{dd}, 2 \mathrm{H}), 4.1(\mathrm{dd}, 1 \mathrm{H}), 6.5-8(\mathrm{~m}, 10 \mathrm{H}, \mathrm{ArH}), 8.2(\mathrm{~s}$, aromatic $\mathrm{OH}),{ }^{13} \mathrm{CNMR}\left(\mathrm{CDCl}_{3}\right) \delta \mathrm{ppm}: 11.7,26.6,37.5,50.8,112.8,115.9,117.0,121.3,127.4,129.1,133.4$, 136.1, 153.4, 156.9,164.5 Mass (m/z): 314. Anal. Calcd. For $\mathrm{C}_{19} \mathrm{H}_{18} \mathrm{~N}_{2} \mathrm{~S}: \mathrm{C}, 68.98 ; \mathrm{H}, 6.11 ; \mathrm{N}, 4.47$. Found: C, 70.02; H, 5.50; N, 4.20.

\section{2-(4'-Chlorophenyl)-4-ethyl-2,3-dihydro-1,5-benzothiazepine (5k)}

IR $(\mathrm{KBr}): v=3040 \mathrm{~cm}^{-1}($ aromatic $-\mathrm{CH})$ 2750, $\left(-\mathrm{CH}_{2} \mathrm{CH}_{3}\right), 1583(\mathrm{C}=\mathrm{N}), 530(\mathrm{C}-\mathrm{Cl}) .{ }^{1} \mathrm{H}$ NMR $\left(\mathrm{CDCl}_{3}\right): \delta \mathrm{ppm}=1.32(\mathrm{~s}, 3 \mathrm{H}), 3.1(\mathrm{dd}, 2 \mathrm{H}), 4.3(\mathrm{dd}, 1 \mathrm{H}), 6.8-8(\mathrm{~m}, 10 \mathrm{H}, \mathrm{ArH}),{ }^{13} \mathrm{CNMR}\left(\mathrm{CDCl}_{3}\right) \delta$ ppm:11.7,26.6,37.7,50.5,112.8, 115.9,117.0,121.3,127.4,125.8, 128.8, 129.1, 132.4, 133.7, 141.6, 151.4.Mass (m/z): 302. Anal. Calcd. For $\mathrm{C}_{19} \mathrm{H}_{18} \mathrm{~N}_{2} \mathrm{~S}$ : C, 67.65; H, 5.34; N, 4.64. Found: C, 66.50; H, $5.20 ; \mathrm{N}, 4.34$. 


\section{Conclusions}

In the current study, we have synthesized an ecofriendly ultrasound assisted synthesis of 2, 4substituted 1, 5- benzothiazepine 5(a-l) with good yield. The remarkable benefits of ultra-sonication as a green synthetic strategy are, reactions were carried at room temperature, required much less time for completion of reaction, highly accelerated reaction rate.

\section{Conflict of interest:}

The authors have declared no conflict of interest.

\section{Acknowledgements:}

The authors are thankful to the Mrs. Fatima Rafiq Zakaria Chairman Maulana Azad Educational Trust, Dr. Maqdoom Farooqui, Principal Maulana Azad Postgraduate \& Research Centre, Aurangabad and Principal, Y.B. Chavan College of Pharmacy, Dr. Rafiq Zakaria Campus, Aurangabad 431001 (M.S.), India for providing the laboratory facility.

\section{Reference:}

1. Mohan, K. K.; Inturi,B.; Pujar, G.V.; Purohit, M.N.; Vijaykumar, G.S.Design, synthesis and 3DQSAR studies of new diphenylamine containing 1,2,4-triazoles as potential anti-tubercular agents. Eur J Med Chem 2014, 84, 516-29.

2. Sumangala, V.; Poojary, B.; Chidananda, N.; Arulmoli, T.; Shenoy, S. Synthesis and biological evaluation of some Schiff bases of 4-amino-5-(4-methylsulfonyl) benzyl-2,4-dihydro-3H-[1,2,4]triazole-3-thione. Med Chem Res. 2013,22,2921-2928.

3. Hunashal, R.D.; Ronad, P.M.; Maddi, V.S.; Satyanarayana, D,; Kamadod, M.A. Synthesis, antiinflammatory and analgesicactivity of 2-[4-(substituted benzylideneamino)-5(substitutedphenoxymethyl)-4H-1,2,4-triazol-3-yl thio] acetic acid deriva-tives. Arab J. Chem 2014, 7, 1070-1778.

4. Vijesh, A.M.; Isloor, A.M.; Shetty, P.; Sundershan, S.; Fun, H.K.; New pyrazole derivatives containing 1,2,4-triazoles and benzoxazoles as potent antimicrobial and analgesic agents. Eur J. Med Chem.2013, 62, 410-415.

5. Miri, R.; Razzaghi-asl, N. and Mohammadi, M. K. "QM study and conformational analysis of an isatin Schiff base as a potential cytotoxic agent," J. of Molecular Modeling, 2013 19, 2, 727-735.

6. Chaubey, A. K.; and Pandeya, S. N. "Synthesis \& anticonvulsant activity (Chemo Shock) of Schiff and Mannich bases of Isatin derivatives with 2-Amino pyridine (mechanism of action)," International J. of PharmTech Research, 2012, 4, 590-598.

7. Wei, D.; Li, N.; Lu, G.; and Yao, K. "Synthesis, catalytic and biological activity of novel dinuclear copper complexwith Schiff base," Science in China B, 2006, 49, 3, 225-229. 
8. Avaji, P. G.; Vinod Kumar, C. H.; Patil, S. A.; Shivananda, K. N.; and Nagaraju, C. "Synthesis, spectral characterization, in-vitro microbiological evaluation and cytotoxic activities of novel macrocyclic bis hydrazone," Eur. J. of Med. Chem., 2009,44, 9, 3552-3559.

9. Wright, A. J. Mayo Clin. Proc., 1999,74, 290.

10. Singh, G.S. Mod Chem appl.,2013,1,3.

11. Ruiz, E.; Rodriguez, H.; Coro, J.; Salfran, E.; Suarez, M.; Martnez-Alvarez, R.; Martin, N.

Ultrason. Sonochem., 2011,18, 32.

12. Safaei-Ghomi, J.; Fadaeian, M.; Hatami, A. Turk. J. Chem., 2007,31,89.

13. Heropoulos, G. A.; Georgakopoulos, S.; Steele, B. R. Tetrahedron Lett., 2005, 46, 2469.

14) Pant S, Pant U, Synthesis of 1,5-benzothiazepines: Part XXXIII-synthesis of antimicrobial studies of 8-substituted 2,5-dihdro-2-(pyridyl)-4-(2-thienyl)1,5-benzothiazepine. Ind J Chem, 2006; 45B:1525-1530

15) Nikalje AP, Vyawahare D, Ghodke M, Green synthesis and pharmacological screening of novel 1,5-benzothiazepines as CNS agents, IJPPS, 2(2), 2010, 27-29.

16) Hideg-Hankovszky et al Chem Abstr, 1971, 49044 e, 75

17) Levai, Albert, Bognar et al, Chem Abstr, 1978; 68:50r, 88 